\title{
Regards
}

\section{Éthique et biodiversité : questions posées à et par la recherche agronomique}

\author{
Pierre Barret ${ }^{1}$, Denis Bourguet ${ }^{2}$, Pierre-Henri Duée ${ }^{3}$, Sophie Gerber ${ }^{4}$, Xavier Le Roux $^{5}$, \\ Michèle Tixier-Boichard ${ }^{6,7}$ \\ 1 Génétique végétale, INRA, UMR GDEC, 63039 Clermont-Ferrand cedex 2, France \\ 2 Écologie et santé des plantes, INRA, UMR CBGP, 34388 Montferrier-sur-Lez, France \\ 3 Physiologie, INRA, Unité Codir, 75338 Paris cedex 07, France \\ 4 Génétique végétale et philosophie, INRA, UMR Biogéco, 33610 Cestas, France \\ 5 Écologie microbienne, INRA, UMR LEM, 69622 Villeurbanne, France \\ 6 Génétique animale, INRA, UMR GABI, 78350 Jouy-en-Josas, France \\ 7 INRA, Direction scientifique Environnement, 75338 Paris cedex 07, France
}

En ce début de XXI ${ }^{\mathrm{e}}$ siècle, des chercheurs de l'Inra s'interrogent sur le sens du développement des technologies en agriculture dans un monde qui remet aujourd'hui en cause une vision dualiste considérant l'homme hors de la nature. NSS se devait d'ouvrir ses pages à cet appel à une éthique de la responsabilité, qui émane de praticiens de la recherche.

La Rédaction

Mots-clés :

agriculture ; responsabilité ; utilitarisme ; biodiversité ; recherche ; agronomie

\section{Keywords:} agriculture; responsibility; user-driven; biodiversity; research; agronomy

\begin{abstract}
Résumé - Les liens entre agriculture et biodiversité posent des questions éthiques à la recherche agronomique. Utilitariste, cette recherche qualifie certaines espèces de nuisibles ou d'utiles et les modifie pour les besoins de l'homme. Pouvons-nous nous autoriser à transformer, contrôler ou éradiquer les espèces qui nous entourent? Les prouesses technologiques peuvent fasciner le chercheur mais offrent une puissance d'intervention qui renforce l'exigence de responsabilité dans la conduite des recherches, vis-à-vis de la nature et de la société. Face à la diversité des écosystèmes naturels, pourquoi un faible nombre de types d'agrosystèmes serait-il la solution? L'agroécologie vise à un fonctionnement mieux intégré entre agriculture et biodiversité pour concilier production et bon état des écosystèmes. Le choix d'un système agricole et de son rapport à la biodiversité révèle le système de valeurs d'une société. Le défi du chercheur est alors de concilier qualité scientifique et conscience réflexive.
\end{abstract}

\begin{abstract}
Ethics and biodiversity: questions to and by agricultural research. Interactions between agriculture and biodiversity raise ethical issues for agricultural research. Being user-driven, agricultural research classifies species as harmful or useful and influences their evolution to meet human needs. Are we allowed to alter, control or destroy species around us? Technological breakthrough may fascinate researchers but the use of increasingly powerful tools intensifies the demand for a responsible conduct of research vis-à-vis both nature and society. Considering the diversity of natural ecosystems, why should a limited number of agricultural systems be the solution? Agroecology aims at a better integration of agriculture and biodiversity in designing systems able to combine production with a healthy status of ecosystems. The choice of an agricultural system and its relationship with biodiversity reveals the values of a society. The challenge for a researcher is thus to combine scientific quality with reflexive consciousness.
\end{abstract}

Auteur correspondant : M. Tixier-Boichard, michele.tixier-boichard@inra.fr 
Le terme «biodiversité » recouvre la diversité des organismes vivants, incluant la diversité génétique au sein de chaque espèce, la diversité des espèces et des écosystèmes, et celle des systèmes socioécologiques. Une large gamme d'interactions entre organismes influence sa dynamique évolutive. Durant les dernières décennies, la biodiversité - dont l'humanité est partie prenante s'est fortement dégradée sous l'effet des activités humaines, de telle sorte que sa protection et même sa restauration sont actuellement l'un des grands enjeux de la recherche (Casetta et Delord, 2014 ; Steffen et al., 2015). Comment reconsidérer les liens entre biodiversité et sociétés humaines? Quelle place tient l'agriculture dans ce contexte ? L'agriculture dite "productiviste», issue de la nécessité de nourrir les populations après la Seconde Guerre mondiale, a conduit à une érosion de la biodiversité et à une simplification des paysages (Le Roux et al., 2008). Cette érosion est dommageable pour l'agriculture elle-même, qui a besoin de préserver la diversité des ressources génétiques, de rechercher et d'utiliser les synergies entre biodiversité et processus de production (pollinisation ; biocontrôle des agresseurs...) afin de concevoir des systèmes agricoles durables.

La recherche agronomique, si elle s'appuie sur les connaissances académiques, est finalisée et anthropocentrée. Elle a pour objectif de comprendre le fonctionnement du vivant pour l'utiliser, le façonner, et " piloter » les systèmes de production. Mais jusqu'où la notion de pilotage peut-elle aller ? Réintégrer la biodiversité dans les processus de production agricole est d'autant plus important que certaines impasses techniques apparaissent, par exemple pour relever les défis imposés par la réduction de l'utilisation de produits phytosanitaires (Jacquet et al., 2011) et par le déclin des pollinisateurs (Goulson et al., 2015).

À l'occasion d'une audition devant le Comité consultatif national d'éthique le 16 octobre 2014, notre groupe de chercheurs (en sciences animales, sciences végétales, microbiologie, écologie et évolution) a exposé les principaux questionnements éthiques rencontrés par chacun dans sa pratique de la recherche agronomique publique. Les réactions du Comité ont alimenté nos réflexions, présentées dans cet article.

\section{Recherche agronomique et utilitarisme}

La recherche agronomique est en grande partie utilitariste, au sens de la satisfaction des besoins de l'homme, puisque sa principale mission est de produire des connaissances utiles permettant notamment à l'agriculture de nourrir les populations humaines.

\section{La recherche agronomique face à la question de l'utilité d'une espèce}

Une espèce jugée « utile » pour l'homme contribue à son bien-être via la fourniture de nourriture, d'énergie ou de travail, par son rôle récréatif ou encore par l'accompagnement qu'elle lui offre dans ses activités. La définition de l'utilité est donc subjective. À l'extrême inverse et de manière tout aussi subjective, une espèce peut être jugée "nuisible » si elle diminue, directement ou indirectement, notre qualité de vie, affecte notre santé ou notre environnement et, en agriculture, réduit les productions quantitatives ou qualitatives des cultures ou des élevages. Les notions d'utile ou de nuisible dépendent ainsi de la vision, patrimoniale ou utilitariste, de la biodiversité. Dans la vision anthropocentrée, contrôler, voire éliminer, une espèce jugée nuisible n'engendre généralement aucun mouvement de protestation, surtout si elle met en danger la vie d'êtres humains. Qui s'est opposé à l'éradication de la variole ? Cette question reste plus ouverte lorsque les espèces considérées ne sont que vectrices d'agents pathogènes: dans le cas des anophèles, qui transmettent le paludisme, les chercheurs apparaissent plus divisés quant au droit de les détruire totalement, si tant est que cela soit possible (Fang, 2010). $\mathrm{Au}$-delà de la question éthique, une telle suppression n'est d'ailleurs pas sans risque car elle peut engendrer des déséquilibres environnementaux importants et peu prévisibles. Par exemple, la prolifération du ver du cotonnier a été contrôlée en Chine grâce à la culture de cotonniers transgéniques, conduisant cependant à l'installation d'un autre insecte nuisible, une punaise $(\mathrm{Wu}$ et al., 2008).

À partir de quel seuil de nuisibilité devons-nous, pouvons-nous éliminer des individus d'une autre espèce, réduire ses populations, les exterminer localement ou de manière définitive, comme dans le cas de la variole? À partir de quel seuil l'acceptons-nous ?

Une vision non utilitariste amène à s'accommoder de l'existence de chaque espèce quel que soit son impact sur les autres et donc sur la nôtre. Toutefois, l'existence d'une espèce est indissociable de ses relations variées avec les autres, allant du mutualisme (coopération, symbiose) aux relations antagonistes (parasitisme, prédation en passant par la compétition). Ces interactions interspécifiques influencent la dynamique des populations et l'évolution des espèces concernées, mais dans le cas de relations antagonistes, on peut admettre qu'il n'y a pas de manipulation lucide, ou consciente, de la part de l'espèce utilisatrice.

La capacité pour l'homme d'être conscient engendret-elle une responsabilité spécifique ? La recherche agronomique, qui a longtemps privilégié, pour la gestion des 
espèces causant des dégâts aux cultures et aux élevages, une approche simplificatrice du type une maladie/un pathogène/une molécule, s'oriente aujourd'hui vers une approche écosystémique plus conforme à la réalité, mais aussi plus compliquée à mener.

\section{La recherche agronomique peut modifier le vivant et affecter son bien-être}

Les objectifs de la sélection végétale ou animale consistent à répondre aux besoins de l'humanité. Jusqu'où peuvent aller, dans ce contexte, les modifications que nous induisons sur la physiologie d'une espèce?

De nombreuses plantes sont totalement tributaires d'une intervention humaine pour leur culture. C'est le cas du maïs, du colza et de la tomate, qui ne sont pas en mesure de se maintenir dans des environnements non anthropisés européens. L'homme a considérablement accru, au cours du temps, le nombre de variétés de ces espèces potentiellement utilisables par l'agriculture, avec, par exemple, plusieurs centaines de variétés de maïs issues de différentes formes de l'ancêtre sauvage, la téosinte.

En sélection animale, l'antagonisme entre fonction de croissance et fonction de reproduction conduit à des situations extrêmes où des lignées sélectionnées (chez la poule ou la dinde) sont si lourdes qu'elles ne peuvent se reproduire sans une intervention humaine. Cette dépendance ne concerne que les génotypes les plus sélectionnés, porteurs d'allèles qui étaient initialement rares, voire absents dans les populations naturelles, car souvent délétères ou incompatibles avec la survie en dehors du milieu contrôlé par l'homme. De même, dans la race bovine blanc bleu belge, la naissance des veaux hypermusclés exige invariablement une césarienne. Cette pratique, acceptée en Belgique, a suscité une forte opposition dans les pays scandinaves où la perception du bien-être animal est différente.

La conception des systèmes de production peut aussi faire l'objet d'un questionnement éthique sur le bien-être animal ; ainsi une densité d'élevage importante provoque des problèmes d'agressivité et prive l'animal de l'expression de ses comportements " ancestraux». Le comité consultatif commun d'éthique pour la recherche agronomique Inra-Cirad (2015) rappelle ainsi que le bien-être animal n'est pas seulement associé à l'absence de souffrance, mais aussi à la prise en compte de la capacité d'un animal à exprimer les comportements naturels de son espèce et à agir selon ses préférences. La distinction peut être faite ici entre souffrance et conscience : par exemple, la claustration des poules pondeuses en cage induit une conscience de contrainte sans qu'une souffrance soit constatée. Des situations paradoxales sont ainsi observées. Les poules pondeuses élevées en plein air, système synonyme de bien-être, présentent des taux de mortalité plus forts que celles logées en bâtiment fermé et en cages aménagées.

La question peut se poser aussi pour les cultures végétales. Ainsi, une vigne doit subir des stress, c'est-àdire être taillée sévèrement, croître sur des sols pauvres et carencés en eau, pour produire des vins de qualité.

La concentration de l'élevage améliore son efficacité économique, mais peut aboutir à des situations extrêmes, telles que l'abattage massif de troupeaux en cas de pathologie contagieuse déclarée dans un périmètre géographique donné. Cette destruction, justifiée par les impacts économiques de la maladie, par exemple le blocage des exportations, peut mettre en danger une race animale et traumatiser les éleveurs, alors que la pathologie, par elle-même, n'induit pas toujours une forte mortalité animale et n'est pas dangereuse pour la santé humaine (exemple de la fièvre aphteuse).

L'être humain s'arroge le droit de modifier le vivant et de lui imposer un mode de reproduction. Prend-il toujours conscience que cette puissance $d$ 'intervention augmente sa responsabilité ?

Il y a bien un questionnement éthique du droit à manipuler, contrôler, modifier, éradiquer les espèces qui nous entourent, pour notre survie, notre subsistance ou notre confort. Cette perception varie cependant en fonction des cultures et des sociétés humaines.

\section{La recherche agronomique : une source d'innovations par et pour la diversité}

\section{La diversification génétique issue de la sélection et son utilisation}

La domestication, puis la sélection, ont façonné la diversité génétique de certaines espèces. Elles ont permis de conserver et de combiner des mutations habituellement éliminées dans la nature, conduisant à l'obtention, par exemple chez les animaux, de nouveaux phénotypes, comme le nanisme ou le gigantisme (chez le chien, par exemple), des colorations variées du pelage ou du plumage... De plus, la sélection a augmenté les performances de croissance, de production (lait, ponte) qui sont maintenant bien supérieures à celles observées chez les ancêtres sauvages, même lorsque ces derniers sont alimentés comme les animaux sélectionnés. L'intervention humaine a ainsi "favorisé » certains génotypes qui auraient été éliminés dans des conditions naturelles. La recherche agronomique contribue alors, de ce point de vue, à la diversification du vivant.

En revanche, le monde agricole, soutenu par la recherche agronomique, privilégie parfois l'utilisation d'un nombre restreint de génotypes (exemple de la race 
Holstein pour la production laitière) avec, pour corollaire, une réduction de la diversité génétique en ferme.

Le cas du blé illustre bien cet antagonisme. Même si l'Inra conserve près de 12000 accessions de blé d'origines géographiques variées, l'augmentation des rendements durant les cinquante dernières années s'est accompagnée d'une érosion progressive de la biodiversité des blés au champ (Bonnin et al., 2014 ; Le Clerc et al., 2006). Cependant, l'intensification de l'agriculture et le choix de cultiver les variétés les plus productives ont libéré ou préservé de l'espace pour d'autres activités humaines (villes, tourisme, loisirs), voire pour la conservation de la nature (forêts et parcs). Il en résulte une spécialisation des territoires, moyen de concilier des objectifs opposés, aux dépens toutefois de la biodiversité des espaces cultivés et de leur environnement proche, avec, par exemple, la chute des populations d'oiseaux communs spécifiques des espaces agricoles et la pollution des nappes phréatiques par les pesticides.

De nombreuses espèces ont été déplacées sous l'action de l'homme. Alors que la dénomination $\mathrm{d}^{\prime}$ « espèce invasive " a souvent une connotation négative, l'introduction volontaire et maîtrisée de nouveaux taxons a été considérée plutôt positivement en agriculture (la pomme de terre, la tomate ou le maïs en Europe). Dans le cas du phylloxéra - un parasite racinaire, apparenté au puceron, destructeur de la vigne cultivée -, l'introduction massive, au début du $\mathrm{XX}^{\mathrm{e}}$ siècle, de portegreffes d'espèces américaines de vigne résistants à cet insecte a sauvé la diversité et la typicité des cépages français!

Le développement de l'agriculture a aussi encouragé la création d'espèces nouvelles, par croisement artificiel entre espèces préexistantes, comme dans le cas du triticale, issu de croisements entre le blé et le seigle, principalement destiné à la production de fourrage.

En élevage, les croisements interspécifiques produisent des animaux aux caractéristiques uniques (mulet, canard mulard), quoique généralement stériles ; toutefois, les croisements entre zébu et vache ont produit des races nouvelles, comme la Borgou d'Afrique de l'Ouest qui a la particularité d'être trypanotolérante. De plus, des croisements inédits se sont produits lors des migrations, pour conduire à des animaux métissés tels que le bovin créole ou le porc créole qui résistent mieux à des climats chauds que les populations européennes dont ils proviennent.

\section{Création de diversité poussée à l'extrême par les biotechnologies}

La mutation est un phénomène naturel à la source de la variabilité génétique et, dans certains cas, phénotypique. La mutagenèse expérimentale (par irradiation ou méthode chimique) produit de la diversité de façon aléatoire, en accélérant la création d'une variabilité qui ne peut être distinguée sur le plan moléculaire de la variabilité naturelle.

La reproduction végétative des plantes cultivées a favorisé le maintien de mutations, plus particulièrement via les transpositions. Ces mutations ont été repérées et conservées par l'homme, alors qu'elles sont généralement éliminées par une phase de reproduction sexuée. Ainsi, les cépages de vignes pinot noir, pinot gris et pinot blanc ont dérivé les uns des autres par des transpositions tout au long de l'histoire de la viticulture (Hocquigny et al., 2004).

Si la création aléatoire de diversité par mutagenèse est une accélération d'un phénomène naturel, les choix importants se situent au moment du criblage. La question des objectifs de sélection se repose : quels cribles, pour quoi faire? Une étape supplémentaire est franchie avec les nouvelles technologies d'édition du génome qui permettent de produire à très grande échelle des gammes d'allèles «à façon » où la modification n'est plus aléatoire, mais déterminée. Il s'agit bien ici d'engendrer, de manière ciblée, de la variabilité.

En quoi cette diversité artificiellement produite serait-elle différente de la diversité naturelle ? Peut-elle représenter un danger? Quels problèmes éthiques sont associés à sa création, mais aussi à son utilisation et son appropriation?

L'information véhiculée par ces deux types de diversité est de nature différente : l'une est issue d'un processus de sélection, de tri, en conditions naturelles ou contrôlées, porteuse d'une information de "réussite » ; l'autre est artificielle, à spectre quasi infini, non issue d'un processus de sélection (en restant compatible toutefois avec la survie et la propagation de l'organismecible dans lequel elle a été induite) et, par conséquent, porteuse d'une information inédite. Ainsi, les biotechnologies permettent de produire des allèles qui n'ont jamais existé, ou qui n'existent plus dans l'ensemble «naturel », et qui pourront aider à répondre aux défis futurs de l'agriculture, mais qui pourraient engendrer des effets inattendus.

L'essor de la transgenèse végétale a induit un fort questionnement éthique, ainsi qu'un intense débat de société. Le transfert naturel de gènes entre espèces est bien décrit chez les bactéries, mais aussi chez les plantes et même chez les animaux, et contribue à l'évolution des espèces. Toutefois, la vitesse d'apparition et l'abondance des variétés transgéniques constituent une accélération de l'action de l'homme sur le vivant, pouvant être source d'inquiétudes et suscitant un fort besoin de régulation. Prend-on le temps de suivre tous les effets sur les écosystèmes cultivés, comme sur les écosystèmes environnants ? La diversité artificielle est-elle porteuse de 
risques nouveaux ou accrus ? Doit-on prendre des précautions particulières dans la façon de mêler des plantes modifiées par biotechnologie et des plantes issues de sélection traditionnelle ou de sélection naturelle?

De plus, l'appropriation du vivant par la brevetabilité choque, que ce soit dans le cas de gènes associés à des procédés comme en Europe, ou dans le cas extrême d'obtentions végétales comme aux États-Unis. Le vivant est alors assimilé à un objet fabriqué et marchand ; l'agriculteur perd alors une partie de son libre arbitre : l'évolution de son métier comme celle de son autonomie sont en jeu.

La biologie de synthèse est un nouvel espace de recherches défini par l'ensemble des actions qui visent à faire fonctionner dans un être vivant, plante ou microorganisme, une nouvelle voie métabolique, préexistante dans un autre être vivant ou pas. Plusieurs résultats ont été obtenus, comme la synthèse de l'artémisinine pour traiter la malaria. On atteint ici un stade ultime du contrôle de l'homme sur la nature. La biologie de synthèse constitue une approche d'ingénierie qui fait resurgir la question du naturel et de l'artificiel, de leur distinction ou de leur confusion. Elle pourrait donner à l'homme la capacité d'inventer de nouvelles espèces, qui deviendraient par essence les premières espèces vivantes artificielles. Cela suscite évidemment de fortes réactions dans la société, de même que des débats entre les chercheurs.

Ainsi, les comités d'éthique, à l'instar de la commission américaine mise en place par le président des ÉtatsUnis en 2010 (Gutmann, 2011), ont approfondi l'analyse des conséquences de ces modifications sur l'évolution du vivant, pour intégrer l'analyse des risques et fournir un cadre de responsabilité à la science. Un tel contexte exige en effet, pour le chercheur, d'expliquer la science qu'il est en train de faire et sa contribution dans la production de connaissances nouvelles et d'innovations.

La puissance de la biotechnologie exercerait-elle une fascination sur les chercheurs ? Peut-on parler d'addiction à la découverte ou de croyance absolue dans la puissance de la technique pour répondre aux besoins d'une population de 10 milliards d'humains en 2050 ? N'y a-til pas d'autres moyens d'atteindre ces objectifs en considérant, plutôt qu'une vision technologique exclusive, la diversité des sociétés humaines et la diversité des solutions qu'elles pourraient trouver pour répondre à leurs besoins, dans leur environnement local et avec la biodiversité qui les entoure? Le questionnement éthique des limites du droit pour l'homme de manipuler et modifier le vivant à sa guise par la prouesse technologique, différemment perçu selon les cultures, est ici reposé.

Cette montée en puissance technologique est le fondement d'une responsabilité nouvelle pour le chercheur, encouragé aussi à explorer les retombées possibles de ses activités. Il devrait ainsi prendre le temps nécessaire à une réflexivité ${ }^{1}$, malgré la pression de la productivité scientifique ou la fascination devant la prouesse technologique.

Le défi posé aux institutions comme l'Inra est donc de penser à concilier qualité scientifique et conscience réflexive, car l'une privée de l'autre, et c'est la recherche publique qui se trouve contrariée dans son ambition de servir l'intérêt général. Ainsi, proposer aux scientifiques une formation à la philosophie des sciences et aux questions d'éthique serait opportun. L'indépendance des travaux menés suppose aussi de partager les questions éthiques avec les partenaires financiers, publics ou privés. Les sociétés savantes internationales ou les grands congrès internationaux peuvent constituer des lieux appropriés pour évoquer ces questions.

\section{La recherche agronomique face aux enjeux de la biodiversité}

$\mathrm{Au} X \mathrm{X}^{\mathrm{e}}$ siècle, l'agriculture s'est développée sans trop se soucier des conséquences de ses pratiques sur les milieux naturels environnants. Aujourd'hui, l'essor de l'agroécologie souligne un intérêt de la recherche agronomique pour l'exploration d'un fonctionnement intégré entre agriculture et biodiversité. Cet essor traduit-il une motivation éthique?

\section{Refonder les relations entre agriculture et biodiversité}

La recherche agronomique, au sein des systèmes agricoles qu'elle étudie, a le devoir d'évaluer les conséquences de ses actions sur la biodiversité, et pas uniquement parce que cette dernière rend des services aux sociétés humaines. Des travaux restent plus que jamais nécessaires pour préciser les relations existant entre biodiversité et agriculture (Le Roux et al., 2008).

Les actions d'une société humaine touchent des sociétés éloignées ; il est donc essentiel de considérer les dommages exportés vers les autres pays. La culture de maïs et de soja imposée par les besoins de l'élevage en Europe et en Asie contribue par exemple à la déforestation en Amazonie. Comment pondérer ces effets ? Ces questions s'insèrent dans le cadre plus large de la réflexion sur les services écosystémiques, définis comme étant les bénéfices que les êtres humains tirent du fonctionnement des écosystèmes (Maris, 2014), et ouvrant un débat interdisciplinaire nouveau.

Si la quantification monétaire de la biodiversité fait débat, elle souligne au moins son importance pour les

\footnotetext{
1 En philosophie, se dit de la conscience qui se prend ellemême pour objet.
} 
sociétés humaines. Son érosion induite par l'agriculture intensive interpelle la recherche agronomique ; elle se doit d'envisager des solutions pour protéger ce bien commun. Mais la notion de services écosystémiques renvoie à une instrumentalisation de la nature et, en incitant à une quantification des bénéfices que l'homme en retire, participe à une dynamique de marchandisation de la biodiversité (Maris, 2014).

Il est par ailleurs primordial d'éviter une vision fixiste de la protection de la biodiversité : maintenir ses capacités d'évolution relève bien de la responsabilité de l'homme vis-à-vis du devenir des espèces naturelles et domestiquées, comme vis-à-vis des générations futures. Une spécialisation de plus en plus poussée des espèces domestiquées et des systèmes de production peut-elle mettre en cause leur capacité d'évolution ? L'homme devrait-il se donner des limites lorsqu'il développe des systèmes de production agricole intensifs, susceptibles de nuire à la diversité du vivant ? Ces systèmes peuvent en effet rencontrer des points de rupture comme celui observé pour les pollinisateurs, qui a motivé un plan gouvernemental aux États-Unis².

\section{L’agriculture révélatrice d'un système de valeurs}

De nouvelles pratiques agricoles sont à inventer pour les agrosystèmes de demain, qui s'appuient sur les synergies entre agriculture et biodiversité dans lesquelles l'humain est partie prenante : il s'agit d'aller vers un anthropocentrisme éclairé, qui sous-tend largement la notion émergente de "solutions fondées sur la nature » (Eggermont et al., 2015). Selon Descola (2011), c'est même en recomposant une écologie des relations entre humains et non-humains que l'anthropologie, acceptant de renoncer à son anthropocentrisme, pourra sortir des débats entre déterminismes naturels et déterminismes culturels.

Le modèle agricole dominant est-il durable ? La recherche a des fonctions d'alerte et de prospective qui peuvent aider à envisager les transformations possibles, sans cependant négliger l'importance que peut prendre le hasard dans l'évolution du vivant. Quelle est la responsabilité de la recherche agronomique publique vis-à-vis des acteurs privés et des pays en développement ? Comment continuer à croître, et le peut-on, dans un monde limité ? Les agronomes sont « convoqués » par les politiques pour garantir l'autosuffisance alimentaire. Face à la diversité des écosystèmes naturels, pourquoi un faible nombre de types d'agrosystèmes serait-il la solution ? La science ne devrait-elle pas plutôt produire les connaissances permettant à une gamme encore plus large de systèmes agricoles de se développer pour répondre aux besoins de différentes populations humaines ? Il s'agit aussi de construire de nouveaux compromis : comment arbitrer l'usage des terres pour tenir compte des nécessités d'urbanisation, soutenir une agriculture permettant une alimentation en quantité et en qualité, accroître les zones forestières ? Ces questions d'arbitrage doivent être appréhendées à l'échelle de la planète, et la gouvernance doit aussi se situer à cette échelle : $c^{\prime}$ est ce qui est attendu de la plateforme intergouvernementale pour la biodiversité et les services écosystémiques ${ }^{3}$ dont le cadre conceptuel mentionne la dimension éthique.

La recherche agronomique et l'agriculture sont donc bien à l'avant-garde des questions touchant à la modification du vivant et des écosystèmes : le choix d'un système agricole est révélateur des besoins d'une société humaine, mais aussi du système de valeurs de cette société et de son positionnement par rapport au monde vivant. La façon dont l'agriculture va évoluer déterminera en grande partie la place de l'homme dans l'environnement et ses relations avec les autres êtres vivants. Cette évolution pose, de façon aiguë, la question de la place de l'éthique dans la pratique du chercheur.

En conclusion, l'accélération des applications technologiques, conduisant parfois à de réelles ruptures, rend inéluctable un questionnement éthique. C'est la responsabilité de l'homme vis-à-vis du monde vivant qui est aujourd'hui engagée. À sa domination et à son exploitation peut se substituer une démarche visant une coévolution, qui force à repenser notre rapport à la nature et à réapprendre le sens de ses limites.

La manière avec laquelle l'homme interagira avec la biodiversité est liée à la façon dont il se définit en tant qu'être humain. Pouvons-nous respecter le monde vivant qui nous entoure, si nous ne nous respectons pas entre nous ? Quel sens donner au « développement durable », ici et maintenant, sachant les inégalités qui s'accroissent sur la planète ? C'est bien l'affirmation $\mathrm{d}$ 'une éthique de responsabilité qui nous amènera à considérer d'autres formes de pérennité de notre développement, jusqu'à inventer une solidarité écologique fondée sur une réconciliation avec la nature, au centre d'un «nouveau contrat social».

La recherche agronomique est attendue dans cette voie, non seulement pour contribuer à résoudre les questions pour demain, mais aussi pour tenter de répondre à celles d'aujourd'hui dans le respect des pratiques culturelles des sociétés et de leurs équilibres de développement, et avec le soin nécessaire au maintien du bon état de notre environnement et des espèces qui y vivent.

\footnotetext{
2 https://www.whitehouse.gov/blog/2015/05/19/ announcing-new-steps-promote-pollinator-health.
} 


\section{Références}

Bonnin I., Bonneuil C., Goffaux R., Montalent P., Goldringer I., 2014. Explaining the decrease in the genetic diversity of wheat in France over the 20th century, Agriculture, Ecosystems and Environment, 195, 183-192.

Casetta E., Delord J. (Eds), 2014. La biodiversité en question. Enjeux philosophiques, éthiques et scientifiques, Paris, Éditions Matériologiques.

Comité consultatif commun d'éthique pour la recherche agronomique Inra-Cirad, 2015. Avis sur le bien-être des animaux d'élevage, Avis 7, Paris, Inra/Cirad, http:// institut.inra.fr/Missions/Promouvoir-ethique-etdeontologie/Avis-du-comite-d-ethique/7e-avis-sur-lebien-etre-des-animaux-d-elevage.

Descola P., 2011. L'écologie des autres. L'anthropologie et la question de la nature. Versailles, Quæ.

Eggermont H., Balian E., Azevedo J.M.N., Beumer V., Brodin T., Claudet J., Fady B., Grube M., Keune H., Lamarque P., Reuter K., Smith M., van Ham C., Weisser W.W., Le Roux $X ., 2015$. Nature-based solutions: new influence for environmental management and research in Europe, GAIA, 24, 243248, doi: 10.14512/gaia.24.4.9.

Fang J., 2010. Ecology: a world without mosquitoes, Nature, 466, 432-434.

Goulson D., Nicholls E., Botías C., Rotheray E.L., 2015. Bee declines driven by combined stress from parasites, pesticides, and lack of flowers, Science, 347, 6229, doi: 10.1126/ science.1255957.

Gutmann A., 2011. The ethics of synthetic biology: guiding principles for emerging technologies, Hastings Center Report, 41, 4, 17-22.
Hocquigny S., Pelsy F., Dumas V., Kindt S., Heloir M., Merdinoglu D., 2004. Diversification within grapevine cultivars goes through chimeric states, Genome, 47, 3, 579-589.

Jacquet F., Butault J.P., Guichard L., 2011. An economic analysis of the possibility of reducing pesticides in French field crops, Ecological Economics, 70, 9, 1638-1648.

Le Clerc V., Cadot V., Canadas M., Lallemand J., Guérin D., Boulineau F., 2006. Indicators to assess temporal genetic diversity in the French Catalogue: no losses for maize and peas, Theoretical and Applied Genetics, 113, 7, 1197-1209.

Le Roux X., Barbault R., Baudry J., Burel F., Doussan I., Garnier E., Herzog F., Lavorel S., Lifran R., Roger-Estrade J., Sarthou J.P., Trometter M. (Eds), 2008. Agriculture and biodiversity. Benefiting from synergies. Multidisciplinary Scientific Assessment. Synthesis report, Paris, Inra http://institut.inra.fr/ en/Objectives/Informing-public-policy/Scientific-ExpertReports/All-the-news/Agriculture-and-Biodiversity. [Une version en français a été publiée par Quæ en 2012 : Agriculture et biodiversité. Valoriser les synergies].

Maris V., 2014. Nature à vendre. Les limites des services écosystémiques, Versailles, Quæ.

Steffen W., Richardson K., Rockström J., Cornell S.E, Fetzer I., Bennett E.M., Biggs R., Carpenter S.R., de Vries W., de Wit C.A., Folke C., Gerten D., Heinke J., Mace G.M., Persson L.M., Ramanathan V., Reyers B., Sörlin S., 2015. Planetary boundaries: guiding human development on a changing planet, Science, 347, 6223, doi:10.1126/science.1259855.

Wu K.M., Lu Y.H., Feng H.Q., Jiang Y.Y., Zhao J.Z., 2008. Suppression of cotton bollworm in multiple crops in China in areas with Bt toxin-containing cotton, Science, 321, 5896, 1676-1678, doi: 10.1126/science.1160550. 